\title{
Presence of zoonotic agents in engorged ticks and hedgehog faeces from Erinaceus europaeus in (sub) urban areas
}

Aleksandra I Krawczyk ${ }^{1 *}$, Arieke Docters van Leeuwen ${ }^{1}$, Wilma Jacobs-Reitsma', Lucas M Wijnands ${ }^{1}$, El Bouw ${ }^{1}$, Setareh Jahfari ${ }^{1,2}$, Angela H A M van Hoek', Joke W B van der Giessen ${ }^{1}$, Jeroen H Roelfsema ${ }^{2}$, Michiel Kroes ${ }^{2}$, Jenny Kleve ${ }^{3}$, Yolanda Dullemont ${ }^{3}$, Hein Sprong ${ }^{1 *}$ and Arnout de Bruin ${ }^{1 *}$

\begin{abstract}
Background: European hedgehogs (Erinaceus europaeus) are hosts for Ixodes hexagonus and I. ricinus ticks, which are vectors for zoonotic microorganisms. In addition, hedgehogs may carry several enteric zoonoses as well. It is unclear to what extent a presence of pathogens in hedgehogs poses a risk to public health, as information on the presence of zoonotic agents in hedgehogs in urban areas is relatively scarce.

Methods: Engorged ticks and hedgehog faeces were collected from rehabilitating hedgehogs. Ticks were screened individually for presence of Borrelia burgdorferi sensu lato, B. miyamotoi, Anaplasma phagocytophilum, and Candidatus Neoehrlichia mikurensis using PCR-based assays. Faecal samples were screened for presence of Campylobacter, Salmonella, Giardia, Cryptosporidium, and extended-spectrum cephalosporin-resistant-Escherichia coli (ESC)-resistant E. coli, using both culture-based and PCR-based methods.

Results: Anaplasma phagocytophilum and Borrelia genospecies B. afzelii, B. spielmanii, B. garinii, and B. burgdorferi sensu stricto were detected in both I. hexagonus and I. ricinus ticks. Despite their widespread distribution in the Netherlands, $B$. miyamotoi and Candidatus N. mikurensis were not detected in collected ticks. Analysis of hedgehog faecal samples revealed the presence of Salmonella enterica subspecies enterica and Campylobacter jejuni. In addition, ESC-resistant E. coli were observed in high prevalence in faecal samples, but no Shiga-toxin producing-E.coli were detected. Finally, potentially zoonotic protozoan parasites were observed in hedgehog faecal samples as well, including Giardia duodenalis assemblage A, Cryptosporidium parvum subtypes IlaA17G1R1 and IICA5G3, and C. hominis subtype IbA10G2.

Conclusions: European hedgehogs in (sub)urban areas harbor a number of zoonotic agents, and therefore may contribute to the spread and transmission of zoonotic diseases. The relatively high prevalence of B. burgdorferi s.l. and $A$. phagocytophilum in engorged ticks, suggests that hedgehogs contribute to their enzootic cycles in (sub)urban areas. To what extent can hedgehogs maintain the enteric zoonotic agents in natural cycles, and the role of (spill-back from) humans remains to be investigated.
\end{abstract}

Keywords: Hedgehogs, Ticks, Zoonoses, Borrelia, Anaplasma, Campylobacter, Salmonella, Antibiotic resistance, Giardia, Cryptosporidium

\footnotetext{
*Correspondence: aleksandra.krawczyk@rivm.nl; hein.sprong@rivm.nl; arnout. de.bruin@rivm.nl

${ }^{1}$ Centre for Zoonoses \& Environmental Microbiology, Centre for Infectious Disease Control, National Institute for Public Health and the Environment, Bilthoven, the Netherlands

Full list of author information is available at the end of the article
} 


\section{Background}

Hedgehogs are host to a wide variety of bacterial and protozoan pathogens [1-3], of which a number have become a matter of concern to public health. Since hedgehogs often dwell in (sub)urban areas, people who rescue or rehabilitate hedgehogs can be exposed to a variety of these pathogens by contact with hedgehogs, their excrements, and vectors. European hedgehogs (Erinaceus europaeus) are a reservoir host for Borrelia burgdorferi sensu lato (Lyme borreliosis), which is widely distributed in the Netherlands, and contributes to maintenance of the bacterium in an enzootic cycle [3,4]. In addition, it was proposed that European hedgehogs are a suitable reservoir host for Anaplasma phagocytophilum, which causes granulocytic anaplasmosis in humans [2]. Both Borrelia genospecies and A. phagocytophilum are transmitted by ixodid ticks, such as Ixodes ricinus that feed on various hosts and $I$. hexagonus that feed predominantly on European hedgehogs [5]. All three life stages of these tick species can feed on humans [6].

In addition to vector-borne agents, hedgehogs are a potential reservoir for enteric bacteria (such as Salmonella and Campylobacter), and protozoan parasites (Giardia and Cryptosporidium), which may cause enteritis in humans, livestock, and pets $[1,7-9]$. The primary transmission route to humans is believed to be food-borne, however, (indirect) contact with an animal reservoir can be an alternative source of infection $[9,10]$. For instance, a study carried out in Denmark reported that strains of Salmonella Enteritidis, isolated from European hedgehogs, belong to the same clonal lineage as strains isolated from infected humans [11].

In contrast, the zoonotic potential of some enteric protozoan parasites has not been fully recognized. Many studies designed to determine genetic groups of protozoan parasites in various hosts, suggest a limited zoonotic potential for Giardia, since strains isolated from people were infrequently found in animals $[12,13]$. Although zoonotic transmission of livestock-associated Cryptosporidium has frequently been described [14], the extent to which wildlife (e.g. hedgehogs) act as a source for Cryptosporidium infection in humans remains unclear.

Finally, little is known about the potential reservoir competence of the European hedgehog for other pathogens transmitted by ixodid ticks, such as Candidatus Neoehrlichia mikurensis, an agent of human neoehrlichiosis, and B. miyamotoi, a recently discovered agent belonging to the relapsing fever group. A number of studies detected Candidatus N. mikurensis in Northern whitebreasted hedgehog (Erinaceus roumanicus) tissue samples in Hungary, and in I. hexagonus feeding on hedgehogs and dogs in the Netherlands and Germany, respectively [15-17]. However, the role of European hedgehogs and their ectoparasites in maintenance of this pathogen in an enzootic cycle is unknown. Borrelia miyamotoi is present in questing I. ricinus in the Netherlands [18], however, it has never been investigated in I. hexagonus before.

In the current study, the presence of a number of zoonotic vector-borne and enteric bacteria and two protozoan parasites was investigated in engorged ticks, obtained from European hedgehogs and hedgehog faeces. In addition, the presence of extended-spectrum cephalosporin (ESC)-resistant E. coli was investigated in faeces, since ESC-resistant E. coli are found in many animal and environmental reservoirs.

\section{Methods}

Collection of Ixodes ticks and DNA extraction procedures Ixodes hexagonus and I. ricinus ticks were collected from European hedgehogs, rehabilitating in a hedgehog shelter in the city of Naarden, and obtained via the Dutch Wildlife Health Centre (DWHC, Utrecht) in 2010, 2011, and 2012. All hedgehogs originated from five different provinces in the Netherlands: Flevoland, Gelderland, Noord-Holland, Utrecht, and Zuid-Holland. In addition, $15 \mathrm{I}$. hexagonus ticks were collected from dead hedgehogs near the city of Ede (province of Gelderland), and from a zoo in the city of Emmen (province of Drenthe) in 2014. Collected samples included ticks of both sexes and all developmental stages with a majority of adult female ticks.

DNA from partially engorged ticks was extracted with ammonium hydroxide as described previously [19]. DNA from fully engorged ticks was extracted using the Qiagen DNeasy Blood \& Tissue Kit according to the manufacturer's protocol for the purification of total DNA from ticks (Qiagen, Venlo, the Netherlands).

\section{Detection of tick-borne pathogens using qPCR, conventional $\mathrm{PCR}$, and sequencing procedures}

Ticks were tested individually for presence of $B$. burgdorferi s.l., B. miyamotoi, A. phagocytophilum and Candidatus N. mikurensis using (q)PCR assays, followed by sequencing for species identification when necessary. For the detection of B. burgdorferi s.l., a duplex real-time PCR was used, based on the detection of fragments of osp $A$ and flagellin genes [20]. A conventional PCR assay, targeting the 5S-23S intergenic region [(IGS) was performed, for Borrelia genospecies identification [21]. Both strands of PCR products were sequenced by BaseClear (Leiden, The Netherlands), using the same forward and reverse primers as in conventional PCR. Borrelia genospecies identification was determined by comparison of sequences to isolates in-house molecular databases (PMID: 23602839). For detection of $B$. miyamotoi, a real-time PCR assay was used that targets a region of the flagellin gene, specific for $B$. miyamotoi [18]. For detection of A. phagocytophilum and Candidatus $\mathrm{N}$. mikurensis a single duplex real-time PCR assay was used that targets a region of the A. phagocytophilum major surface protein $(m s p 2)$ gene [22], and a region specific for 
Candidatus N. mikurensis of the heat shock protein gene groEL [17]. Due to limitations of available DNA, not all ticks $(n=628)$ were tested for all pathogens. For numbers of ticks tested for each vector-borne pathogen, see Table 1.

\section{Collection of hedgehog faeces, DNA extraction, detection of enteric pathogens, protozoan parasites, and anti- microbial resistance genes}

No ethical approval is required for the experimental methods used in this study. The hedgehog shelter has a permit for handling and rehabilitating hedgehogs by the State Secretary for Economic Affairs, Agriculture and Innovation, according article 75 of the Dutch 'Animal Health and Welfare Act'. Hedgehog faeces were collected from 90 hedgehogs, rehabilitating in the hedgehog shelter in the city of Naarden in April $(\mathrm{n}=58)$ and October $(n=32)$ of 2013 . Hedgehogs originated from five different provinces in the Netherlands, described before, and were brought to the shelter due to apparent sickness or injury. Hedgehog faecal material was examined for the presence of Campylobacter by standard microbiological methods, according to ISO/DIS 10272-1 [23]. Confirmation was based on typical microscopic appearance of suspect colonies on mCCDA plates, and by PCR in order to distinguish between C. coli, C. jejuni, C. lari and C. upsaliensis isolates [24].

Faecal samples were also tested for the presence of Salmonella according to Annex D of ISO 6579 [25], and Shiga toxin-producing E. coli according to ISO/TS 13136 [26]. After the presence of Salmonella was confirmed, serotyping was performed using the method of Grimont and Weill [27].

Expanded spectrum cephalosporin-resistant E. coli (ESC-resistant E. coli) were isolated by direct streaking of a loop $(10 \mu \mathrm{l})$ of hedgehog faeces on Brilliance $E$. coli/ coliform Selective Agar (Oxoid), supplemented with

Table 1 Prevalence of tick-borne pathogens in ticks feeding on hedgehogs

\begin{tabular}{lllll}
\hline & \multicolumn{2}{l}{ Ixodes hexagonus } & \multicolumn{2}{l}{ Ixodes ricinus } \\
\cline { 2 - 5 } & $\mathbf{n}$ & $\%$ & $\mathbf{n}$ & $\%$ \\
\hline B. burgdorferi s.l. & $60 / 435$ & 14 & $7 / 25$ & 28 \\
B. afzelii & $37 / 49$ & 76 & n.d. & - \\
B. garinii & $3 / 49$ & 6 & n.d. & - \\
B. spielmani & $7 / 49$ & 14 & n.d. & - \\
B. burgdorferi s.s. & $2 / 49$ & 4 & n.d. & - \\
B. miyamotoi & $0 / 170$ & 0 & $0 / 25$ & 0 \\
A. phagocytophilum & $74 / 277$ & 27 & $6 / 25$ & 24 \\
Candidatus N. mikurensis** & $0 / 251$ & 0 & $0 / 25$ & 0 \\
\hline
\end{tabular}

n.d. $=$ not determined.

*Our results (30/84) were compiled with data obtained from [40], where 44/193 I. hexagonus ticks were found positive for A. phagocytophilum. **Our results $(0 / 84)$ were compiled with data from [17], where none of the 167 tested I. hexagonus ticks were found positive for Candidatus N. mikurensis.
$1 \mu \mathrm{g} / \mathrm{ml}$ cefotaxime (Sigma). Suspected ESC-resistant $E$. coli were phenotypically confirmed with a combination disc-diffusion test according to CLSI guidelines [28]. Cefotaxime and ceftazidime discs, with and without clavulanic acid, were used to identify ESBL-producing $E$. coli. A cefoxitin disc was used to detect isolates with an AmpC phenotype.

For detection of protozoan parasites, DNA was isolated from faecal samples using the High Pure PCR template DNA isolation kit from Roche (Almere, The Netherlands), according to the manufacturer's instructions. Detection of Giardia duodenalis, Cryptosporidium parvum, and C. hominis was performed using a multiplex real-time PCR [29]. Molecular typing of Cryptosporidium species was performed by sequencing an amplified fragment of the GP60 gene [30]. The assemblage of G. duodenalis was established using a PCR on marker 4E1-HP, specific for either assemblage A or $B$ [31], which are associated with human infections.

\section{Results and discussion}

Regarding tick-borne pathogens, we detected B. burgdorferi s.l. in $14 \%(60 / 435)$ of $I$. hexagonus ticks and $28 \%(7 / 25)$ of I. ricinus ticks feeding on European hedgehogs (Table 1). Intergenic spacer (IGS) sequencing of 49 PCR-positive $I$. hexagonus ticks revealed several known Borrelia genospecies: B. afzelii (76\%), B. spielmanii (14\%), B. garinii (6\%), and $B$. burgdorferi s.s. (4\%; Table 1$)$. These findings are consistent with previous studies, which revealed the presence of the same Borrelia genospecies in ticks feeding on hedgehogs in Germany and Switzerland [3,4]. This suggests that the European hedgehog may be a reservoir host for B. burgdorferi s.l. also in the Netherlands as, and may influence local Lyme borreliosis risk.

In addition to B. burgdorferi s.l. genospecies, A. phagocytophilum was detected as well in Ixodes ticks feeding on European hedgehogs. DNA was detected in 27\% (68/ $251)$ of $I$. hexagonus ticks and in $24 \%(6 / 25)$ of $I$. ricinus ticks (Table 1). The relatively high prevalence of $A$. phagocytophilum found in the current study supports the idea, proposed by other researchers, that E. europaeus is a reservoir host for this pathogen $[2,4,32]$.

Ixodes hexagonus as a nidicolous species, rarely bites humans and its direct epidemiological importance is unknown [5,6]. However, it seems to contribute to the circulation of both B. burgdorferi s.l., and A. phagocytophilum in nature $[2,33]$. In addition its predominant host, E. europaeus, may harbour all life stages of generalist $I$. ricinus ticks, which successfully infect hedgehogs with at least one major group of zoonotic agents: $B$. burgdorferi s.l. [34]. In certain habitats, hedgehogs may be the main host for I. ricinus ticks, which may acquire pathogens via either co-feeding or systemic transmission [35]. Subsequently, I. ricinus ticks may transmit a number of bacterial pathogens (e.g. A. phagocytophilum 
and B. burgdorfei s.l.) to other vertebrates as well, including humans.

To our knowledge, this is the first study that has tested ticks feeding on hedgehogs for B. miyamotoi, a spirochete belonging to the relapsing fever group. The absence of this pathogen in $I$. hexagonus may indicate that this specialist tick species is not a competent vector, or that E. europaeus is not a competent host for B. miyamotoi. However, it was shown that $4 \%$ of questing $I$. ricinus ticks were positive for B. miyamotoi in the Netherlands [36]. Therefore, it is also possible that the number of investigated I. ricinus ticks in the current study was not sufficient to detect this bacterium.

Finally, no Candidatus N. mikurensis DNA was detected in either I. ricinus or I. hexagonus ticks feeding on $E$. europaeus. This finding is consistent with another study, in which this pathogen was also not detected in $I$. hexagonus feeding on Dutch hedgehogs [17]. Human and animal cases of Candidatus N. mikurensis infections have (as of yet) not been reported in the Netherlands, and the prevalence of this pathogen in questing I. ricinus ticks is relatively low [17]. Therefore, it is still unclear whether this pathogen may pose risk to public health in the Netherlands.

We detected Salmonella in 10\% (9/90) of hedgehog faecal samples (Table 2). Salmonellosis is a zoonosis that has already been associated with hedgehogs, including E. europaeus. Several studies reported at least three different serotypes in hedgehogs that are pathogenic to humans: Salmonella Tilene, Salmonella Typhymurium and Salmonella Enteritidis [1,11]. Three isolates obtained from these faecal samples were characterized as Salmonella enterica subsp. enterica serotype Enteritidis, which is a common serotype pathogenic to humans [37].

One faecal sample (1\%) contained Campylobacter, which is the second most common food-borne bacterium worldwide [10]. Further genotyping revealed $C$. jejuni, which is recognized as one of the main causes of human gastroenteritis. A study in Denmark also reported the presence of

\section{Table 2 Prevalence of enteric pathogenic bacteria, protozoan parasites, and ESC-resistant $E$. coli in hedgehog faeces}

\begin{tabular}{lllllll}
\hline & \multicolumn{7}{l}{ April $\mathbf{2 0 1 3}$} & \multicolumn{2}{l}{ October } & $\mathbf{2 0 1 3}$ & Total \\
\cline { 2 - 7 } & $\mathbf{n = 5 8}$ & $\mathbf{\%}$ & $\mathbf{n}=\mathbf{3 2}$ & $\mathbf{\%}$ & $\mathbf{n = 9 0}$ & $\mathbf{\%}$ \\
\hline Salmonella spp. & 1 & 2 & 8 & 25 & 9 & 10 \\
Campylobacter spp. & 0 & 0 & 1 & 3 & 1 & 1 \\
Shiga toxin-producing E.coli & 0 & 0 & 0 & 0 & 0 & 0 \\
Giardia spp. & 3 & 5 & 7 & 22 & 10 & 11 \\
Cryptosporidium spp. & 3 & 5 & 5 & 16 & 8 & 9 \\
ESC-resistant E. coli & 51 & 88 & 13 & 41 & 64 & 71 \\
AmpC-producing E. coli & 50 & 86 & 0 & 0 & 50 & 56 \\
ESBL-producing E. coli & 1 & 2 & 13 & 41 & 14 & 16 \\
\hline
\end{tabular}

C. jejuni in hedgehogs, which were rehabilitating in private homes, or fed in gardens [9]. No Shiga toxin-producing E.coli were detected in the faecal samples investigated.

In addition to zoonotic enteric bacteria, 11\% (10/90) of hedgehog faecal samples were positive for Giardia species (Table 2). Giardia is a genus of flagellated protozoan parasites divided into eight major genetic groups $(\mathrm{A}-\mathrm{H})$ called assemblages, which slightly differ in morphology and may cause disease in diverse vertebrate hosts [13]. We detected G. duodenalis assemblage A in hedgehog faecal samples, which is responsible for human infections worldwide [12]. However, data regarding the presence of Giardia in hedgehogs are scarce, and until now no Giardia assemblages were found associated with these animals.

We detected Cryptosporidium species in 9\% (8/90) of hedgehog faecal samples as well. Cryptosporidium is another genus of protozoan parasites of vertebrates, which causes enteric infections in humans. In this study, two genospecies, C. parvum (subtype: IIaA17G1R1 and IIcA5G3) and $C$. hominis (subtype: IbA10G2) were observed. These subtypes cause the majority of cryptosporidiosis in humans [14]. Cryptosporidium hominis subtype Ib is primarily transmitted anthroponotically and, to the best of our knowledge, it has never been detected in hedgehog faeces before. In addition, C. parvum subtype IIaA17G1R1 has never been detected in these animals either, but was described in calves, which may play a role in the transmission of human cryptosporidiosis [38]. Interestingly, subtype IIcA5G3, which is considered to be human specific, has been isolated from hedgehog faeces previously [7]. The presence of those subtypes in hedgehog faeces may indicate transmission of the pathogen within hedgehog populations, as suggested before [7].

Finally, viable ESC-resistant E. coli were detected in 71\% (64/90) of hedgehog faecal samples (Table 2). AmpCproducing $E$. coli were only found in the samples collected in April, but at a high prevalence of $86 \%(50 / 58)$. A lower prevalence of 41\% (13/32) of ESBL-producing E.coli was observed in samples collected in October. Only one isolate collected in April had the same phenotype. To the best of our knowledge this is the first description of ESC-resistant E. coli in hedgehogs. In the literature, an ESBL-producing isolate from hedgehog faeces was reported, however, this isolate was later identified as a Klebsiella pneumoniaea strain [39]. The relatively high prevalence of ESC-resistant E. coli in tested hedgehog faeces, especially in the April samples is intriguing. If this might pose a risk to humans, handling rehabilitating hedgehogs has to be investigated. It is very likely that these enteric pathogens, protozoan parasites, and ESC-resistant E. coli detected in hedgehog faecal samples were acquired by ingestion of contaminated materials found in the habitat of hedgehogs, and originates from other animals or humans (waste, food, etc.). 


\section{Conclusions}

Although hedgehog blood or tissue samples were not available for examination, a relatively high prevalence of vector-borne pathogens $B$. burgdorferi s.l. genospecies and $A$. phagocytophilum in engorged ticks obtained from $E$. europaeus, indicates that hedgehogs contribute to pathogen maintenance in natural cycles in (sub)urban areas in the Netherlands.

A number of enteric pathogenic bacteria, protozoan parasites, and ESC-resistant E. coli are present in faecal material, obtained from Dutch E. europaeus. This may pose a risk for people handling diseased and wounded animals, because they can come into contact with contaminated hedgehog faeces. However, to understand the transmission of infection between wildlife and humans, a thorough understanding of the population genetics of pathogens and hosts is required. To investigate this issue more in depth, isolates obtained from wildlife should be compared with human isolates, which represent serotypes that are epidemiologically important with regard to public health.

\section{Competing interests}

The authors declare that they have no competing interests.

\begin{abstract}
Authors' contributions
AK and AdB analyzed data, and wrote the final manuscript. JK and YD collected engorged ticks from hedgehogs and hedgehog faeces. ADVL and SJ performed laboratory tests and analyzed laboratory results on all data obtained for vector-borne pathogens. SJ provided additional data as well on A. phagoctyophilum and Candidatus Neoehrlichia mikurensis. Laboratory tests and analyses of results regarding enteritic pathogens were performed by WJR (Campylobacter), LW (Salmonella), and EB (Shiga toxin-producing E. coli). Laboratory tests and data analyses regarding ESC-resistant E. coli were performed by $\mathrm{AvH}$. Laboratory tests and data analyses regarding protozoan parasites were performed by JR (Giardia), and MK (Cryptosporidium). HS designed and supervised the study. All authors read and approved the final manuscript.
\end{abstract}

\section{Acknowledgments}

We would like to thank the general public for bringing injured or sick hedgehogs to the hedgehog shelter in Naarden. In addition, we would like to thank all volunteers of the hedgehog shelter in Naarden for taking care of the animals and sample collection. This study was financially supported by the Dutch Food and Consumer Product Safety Authority (NWWA) and by the Ministry of Health, Welfare and Sport (WWS). This work was done under the frame of EurNegVec Cost Action TD1303.

\section{Author details}

${ }^{1}$ Centre for Zoonoses \& Environmental Microbiology, Centre for Infectious Disease Control, National Institute for Public Health and the Environment, Bilthoven, the Netherlands. ${ }^{2}$ Centre for Research Infectious Diseases Diagnostics and Screening, Centre for Infectious Disease Control, National Institute for Public Health and the Environment, Bilthoven, the Netherlands. ${ }^{3}$ Stichting Egelbescherming Nederland, Naarden, the Netherlands.

\section{Received: 5 February 2015 Accepted: 18 March 2015}

Published online: 09 April 2015

\section{References}

1. Riley PY, Chomel BB. Hedgehog zoonoses. Emerg Infect Dis. 2005;11(1):1-5.

2. Silaghi C, Skuballa J, Thiel C, Pfister K, Petney T, Pfaffle M, et al. The European hedgehog (Erinaceus europaeus)-a suitable reservoir for variants of Anaplasma phagocytophilum? Ticks Tick Borne Dis. 2012;3(1):49-54.
3. Gern L, Rouvinez E, Toutoungi LN, Godfroid E. Transmission cycles of Borrelia burgdorferi sensu lato involving Ixodes ricinus and/or I. hexagonus ticks and the European hedgehog, Erinaceus europaeus, in suburban and urban areas in Switzerland. Folia Parasitol (Praha). 1997;44(4):309-14.

4. Skuballa J, Oehme R, Hartelt K, Petney T, Bucher T, Kimmig P, et al. European Hedgehogs as Hosts for Borrelia spp., Germany. Emerg Infect Dis. 2007;13(6):952-3.

5. Pfaffle M, Petney T, Skuballa J, Taraschewski H. Comparative population dynamics of a generalist (Ixodes ricinus) and specialist tick (I. hexagonus) species from European hedgehogs. Exp Appl Acarol. 2011;54(2):151-64.

6. Estrada-Pena A, Jongejan F. Ticks feeding on humans: a review of records on human-biting Ixodoidea with special reference to pathogen transmission. Exp Appl Acarol. 1999;23(9):685-715.

7. Dyachenko V, Kuhnert Y, Schmaeschke R, Etzold M, Pantchev N, Daugschies A. Occurrence and molecular characterization of Cryptosporidium spp. genotypes in European hedgehogs (Erinaceus europaeus L.) in Germany. Parasitology. 2010;137(2):205-16.

8. Chilvers BL, Cowan PE, Waddington DC, Kelly PJ, Brown TJ. The prevalence of infection of Giardia spp. and Cryptosporidium spp. in wild animals on farmland, southeastern North Island, New Zealand. Int J Environ Health Res. 1998:8(1):59-64.

9. Petersen L, Nielsen EM, Engberg J, On SL, Dietz HH. Comparison of genotypes and serotypes of Campylobacter jejuni isolated from Danish wild mammals and birds and from broiler flocks and humans. Appl Environ Microbiol. 2001;67(7):3115-21.

10. Sahin O, Fitzgerald C, Stroika S, Zhao S, Sippy RJ, Kwan P, et al. Molecular evidence for zoonotic transmission of an emergent, highly pathogenic Campylobacter jejuni clone in the United States. J Clin Microbiol. 2012;50(3):680-7.

11. Nauerby B, Pedersen K, Dietz HH, Madsen M. Comparison of Danish isolates of Salmonella enterica serovar enteritidis PT9a and PT11 from hedgehogs (Erinaceus europaeus) and humans by plasmid profiling and pulsed-field gel electrophoresis. J Clin Microbiol. 2000;38(10):3631-5.

12. Sprong H, Caccio SM, van der Giessen JW, network Z, partners. Identification of zoonotic genotypes of Giardia duodenalis. PLoS Negl Trop Dis. 2009;3(12):e558.

13. Ryan U, Caccio SM. Zoonotic potential of Giardia. Int J Parasitol. 2013;43(12-13):943-56.

14. Monis PT, Thompson RC. Cryptosporidium and Giardia-zoonoses: fact or fiction? Infect Genet Evol. 2003;3(4):233-44.

15. Krucken J, Schreiber C, Maaz D, Kohn M, Demeler J, Beck S, et al. A novel highresolution melt PCR assay discriminates Anaplasma phagocytophilum and "Candidatus Neoehrlichia mikurensis". J Clin Microbiol. 2013;51(6):1958-61.

16. Foldvari G, Jahfari S, Rigo K, Jablonszky M, Szekeres S, Majoros G, et al. Candidatus Neoehrlichia mikurensis and Anaplasma phagocytophilum in urban hedgehogs. Emerg Infect Dis. 2014;20(3):496-8.

17. Jahfari S, Fonville M, Hengeveld P, Reusken C, Scholte EJ, Takken W, et al. Prevalence of Neoehrlichia mikurensis in ticks and rodents from North-west Europe. Parasites \& vectors. 2012;5:74.

18. Hovius JW, de Wever B, Sohne M, Brouwer MC, Coumou J, Wagemakers A et al. A case of meningoencephalitis by the relapsing fever spirochaete Borrelia miyamotoi in Europe. Lancet. 2013;382(9892):658.

19. Wielinga PR, Gaasenbeek C, Fonville M, de Boer A, de Vries A, Dimmers W, et al. Longitudinal analysis of tick densities and Borrelia, Anaplasma, and Ehrlichia infections of Ixodes ricinus ticks in different habitat areas in The Netherlands. Appl Environ Microbiol. 2006;72(12):7594-601.

20. Heylen D, Tijsse E, Fonville M, Matthysen E, Sprong H. Transmission dynamics of Borrelia burgdorferi s.l. in a bird tick community. Environ Microbiol. 2013;15(2):663-73.

21. Coipan EC, Jahfari S, Fonville M, Maassen CB, van der Giessen J, Takken W, et al. Spatiotemporal dynamics of emerging pathogens in questing Ixodes ricinus. Front Cell Infect Microbiol. 2013;3:36.

22. Courtney JW, Kostelnik LM, Zeidner NS, Massung RF. Multiplex real-time PCR for detection of anaplasma phagocytophilum and Borrelia burgdorferi. J Clin Microbiol. 2004;42(7):3164-8.

23. Anonymous. Microbiology of the food chain - Horizontal method for detection and enumeration of Campylobacter- Part 1: Detection method. ISO/DIS 10272-1. 2015.

24. Jensen AN, Andersen MT, Dalsgaard A, Baggesen DL, Nielsen EM. Development of real-time PCR and hybridization methods for detection and identification of thermophilic Campylobacter spp. in pig faecal samples. J Appl Microbiol. 2005;99(2):292-300. 
25. Anonymous. Amd1 Annex D: Detection of Salmonella spp. in animal faeces and in environmental samples from the primary production stage. International Organization for Standardisation, Geneva, Switzerland. ISO 6579. 2002.

26. Anonymous. Microbiology of food and animal feed - Real-time polymerase chain reaction (PCR)-based method for the detection of food-borne pathogens - Horizontal method for the detection of Shiga toxin-producing Escherichia coli (STEC) and the determination of 0157, 0111, 026, 0103 and 0145 serogroups. ISO/TS 13136. 2012.

27. Grimont PAD, Weill FX. Antigenic formulae of the Salmonella serovars. 9th ed. Institute Pasteur, Paris, France: WHO Collaborating Centre for Reference and Research on Salmonella; 2007.

28. Anonymous. CLSI: Performance Standards for Antimicrobial Susceptibility Testing: Twenty-first Informational Supplement M100-21. Wayne, PA, USA. 2011.

29. Verweij JJ, Blange RA, Templeton K, Schinkel J, Brienen EA, van Rooyen MA, et al. Simultaneous detection of Entamoeba histolytica, Giardia lamblia, and Cryptosporidium parvum in fecal samples by using multiplex real-time PCR. J Clin Microbiol. 2004;42(3):1220-3.

30. Strong WB, Gut J, Nelson RG. Cloning and sequence analysis of a highly polymorphic Cryptosporidium parvum gene encoding a 60-kilodalton glycoprotein and characterization of its 15- and 45-kilodalton zoite surface antigen products. Infect Immun. 2000;68(7):4117-34.

31. Vanni I, Caccio SM, van Lith L, Lebbad M, Svard SG, Pozio E, et al. Detection of Giardia duodenalis assemblages A and B in human feces by simple, assemblage-specific PCR assays. PLoS Negl Trop Dis. 2012;6(8):e1776.

32. Nijhof AM, Bodaan C, Postigo M, Nieuwenhuijs H, Opsteegh M, Franssen L, et al. Ticks and associated pathogens collected from domestic animals in the Netherlands. Vector Borne Zoonotic Dis. 2007;7(4):585-95.

33. Estrada-Pena A, Oteo JA, Estrada-Pena R, Gortazar C, Osacar JJ, Moreno JA, et al. Borrelia burgdorferi sensu lato in ticks (Acari: Ixodidae) from two different foci in Spain. Exp Appl Acarol. 1995;19(3):173-80.

34. Gray JS, Kahl O, Janetzki-Mittman C, Stein J, Guy E. Acquisition of Borrelia burgdorferi by Ixodes ricinus ticks fed on the European hedgehog, Erinaceus europaeus L. Exp Appl Acarol. 1994;18(8):485-91.

35. Randolph SE, Gern L, Nuttall PA. Co-feeding ticks: Epidemiological significance for tick-borne pathogen transmission. Parasitol Today. 1996;12(12):472-9.

36. Fonville M, Friesema IH, Hengeveld PD, van Docters Leeuwen A, Jahfari S, Harms MG, et al. Human exposure to tickborne relapsing fever spirochete Borrelia miyamotoi, the Netherlands. Emerg Infect Dis. 2014;20(7):1244-5.

37. Baumler AJ, Hargis BM, Tsolis RM. Tracing the origins of Salmonella outbreaks. Science. 2000;287(5450):50-2.

38. Li N, Xiao L, Cama VA, Ortega Y, Gilman RH, Guo M, et al. Genetic recombination and Cryptosporidium hominis virulent subtype IbA10G2. Emerg Infect Dis. 2013;19(10):1573-82.

39. Poirel L, Nordmann P, Ducroz S, Boulouis HJ, Arne P, Millemann Y. Extended-spectrum beta-lactamase CTX-M-15-producing Klebsiella pneumoniae of sequence type ST274 in companion animals. Antimicrob Agents Chemother. 2013;57(5):2372-5.

40. Jahfari $S$, Coipan EC, Fonville $M$, van Leeuwen AD, Hengeveld P, Heylen D, et al. Circulation of four Anaplasma phagocytophilum ecotypes in Europe. Parasites \& vectors. 2014;7:365.

\section{Submit your next manuscript to BioMed Central and take full advantage of:}

- Convenient online submission

- Thorough peer review

- No space constraints or color figure charges

- Immediate publication on acceptance

- Inclusion in PubMed, CAS, Scopus and Google Scholar

- Research which is freely available for redistribution 